\title{
A Quantitative Study of Retrograde Axonal Transport in Motor and Sensory Neurons
}

\author{
DAVID J. FINK, ${ }^{1}$ DAVID PURKISS AND MARINA MATA \\ Neurology Research Laboratory, University of Michigan and VA Medical Center, Ann Arbor, MI 48105
}

Received 27 December 1985

\begin{abstract}
FINK, D. J., D. PURKISS AND M. MATA. A quantitative study of retrograde axonal transport in motor and sensory neurons. BRAIN RES BULL 16(4) 537-540, 1986.- We used ${ }^{3} \mathrm{H}$ N-succinimidyl propionate to covalently label in vivo proteins of the rat sciatic nerve, and studied the accumulation of radioactively labeled proteins in the cell bodies of the ipsilateral dorsal root ganglion and ventral horn of spinal cord to assess retrograde axonal transport in sensory and motor neurons respectively. In each case the early accumulation of a small amount of radioactively labeled protein is followed by the later accumulation of a larger amount, which subsequently declines to lower levels. The differences between accumulation in the motor neuron and sensory neuron are discussed. Quantitative assessment of retrograde axonal transport will allow future determination of alterations in that transport after nerve injury and in toxic states, which will help elucidate the role of retrogradely transported proteins in neuronal cell biology.
\end{abstract}

Retrograde axonal transport $\quad{ }^{3} \mathrm{H}$ N-Succinimidyl propionate $\quad$ Peripheral nerve Autoradiography

${ }^{3} \mathrm{H}$ N-SUCCINIMIDYL PROPIONATE $\left({ }^{3} \mathrm{H}\right.$ NSP) is an acylating reagent which may be used to covalently radioactively label endogenous axonal proteins in vivo $[2,14]$. We have previously used this agent to study slow anterograde axonal transport and to define slow retrograde axonal transport in the sciatic nerve of rats, but we were unable to detect rapid transport in either direction in those studies $[5,6]$.

Because rapid retrograde transport from the axon back towards the cell body is generally considered to provide feedback to the cell body about the state of the axon and to play a critical role in maintaining neuronal functional integrity $[1,3,8]$, we were interested in investigating the dynamics of rapid retrograde transport in the sciatic nerve. In the neurosecretory neurons of the neurohypophysis we previously demonstrated ${ }^{3} \mathrm{H}$ NSP labeling of rapid anterograde transport by the accumulation of radiolabeled transported proteins in the collected nerve terminals that comprise the posterior pituitary [4]. Similarly, rapid retrograde transport has been demonstrated using ${ }^{3} \mathrm{H}$ NSP in biochemical studies of the goldfish optic nerve [15] and autoradiographic studies of central nervous system pathways in the rat [9].

We undertook the current study to determine whether accumulation over time of more rapidly retrogradely transported proteins could be detected in the ccll bodies of motor and sensory neurons and whether the amount of that transport could be defined quantitatively.
METHOD

\section{Animal Injections}

Malc Sprague Dawley rats (200-250 grams) were used in all experiments. The animals were anesthetized with chloral hydrate and the sciatic nerve exposed in the gluteal region. ${ }^{3} \mathrm{H}$ NSP $(50 \mathrm{Ci} / \mathrm{mmol}$ in benzene, New England Nuclear) was evaporated to dryness under vacuum on ice and resuspended in 3 microliters of physiologic saline, $\mathrm{pH}$ 7.4. One microliter was injected subepineurially over 5 minutes using a 30 gauge needle attached to a Hamilton syringe in a Harvard infusion pump and the wound closed with wound clips.

At selected times $(1,2,7,14,21$ and 28 days) after injection the animals were again anesthetized with chloral hydrate and sacrificed by perfusion through the heart with $0.1 \mathrm{M}$ phosphate buffered saline. The injected sciatic nerve and bilateral L4, L5 and L6 dorsal root ganglia (DRG) were removed, placed in $5 \mathrm{mM}$ Tris- $\mathrm{HCl}$ saline, $\mathrm{pH} 7.4$ and both the nerve samples and the pooled ganglia from each side were precipitated with $10 \%$ trichloroacetic acid, washed twice with acetone and twice with ether, and the final pellet suspended in 50 microliters of buffer containing $1 \%$ SDS and $1 \%$ beta mercaptoethanol. After heating to 95 degrees $\mathrm{C}$ for 3 minutes duplicate aliquots were taken for determination of radioactivity in an LKB 1218 beta counter. The spinal cord was removed en bloc, frozen, and 200 micron sections cut on an IEC cryostat at $\mathbf{- 8}$ degrees $\mathrm{C}$. The ipsilateral and con-

\footnotetext{
'Requests for reprints should be addressed to David J. Fink, Neurology (127), 2215 Fuller Road, Ann Arbor, MI 48105.
} 


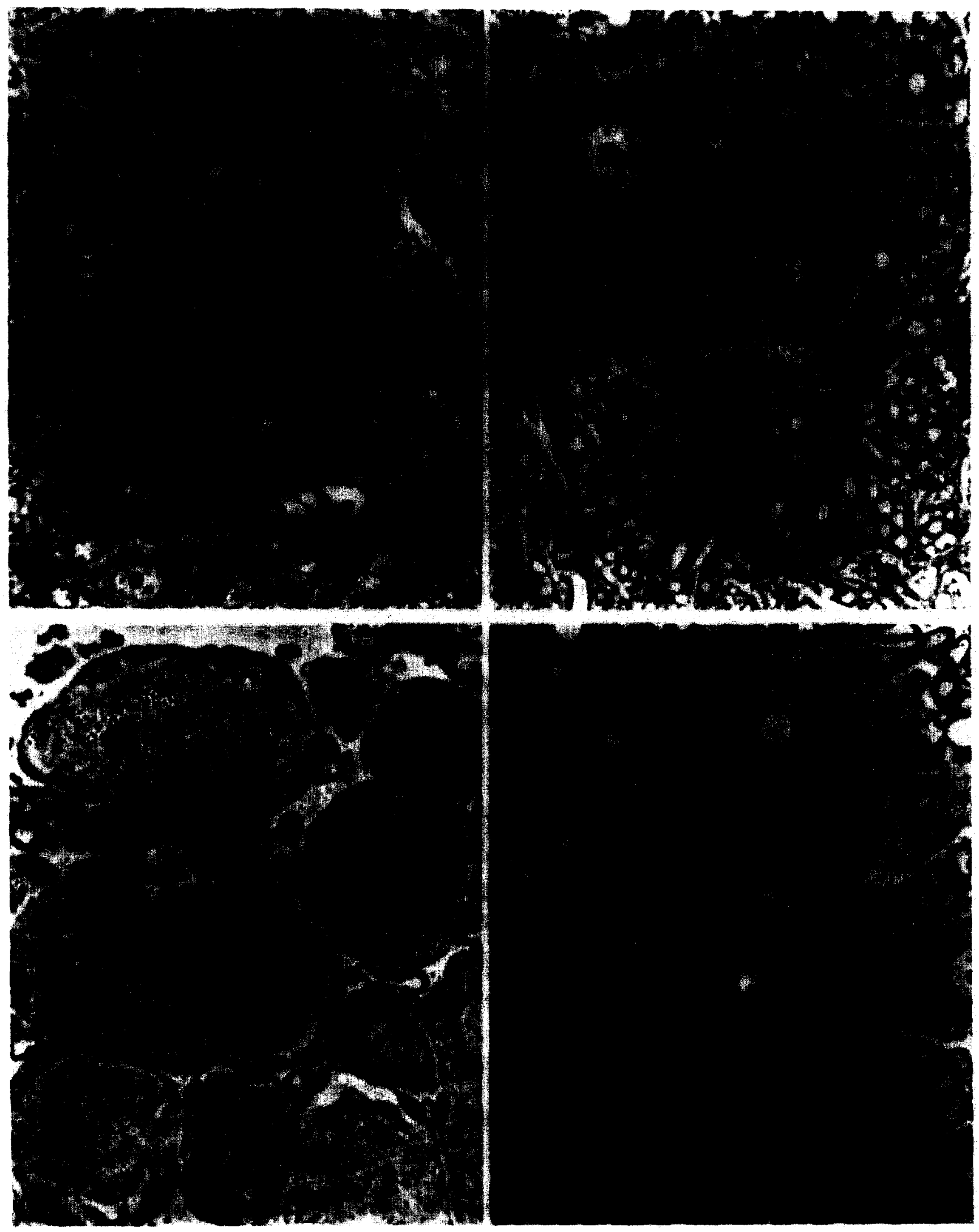


tralateral ventral horns in the region of the lumbar enlargement were removed using a 750 micron diameter punch. These samples were processed as described above.

The amount of radioactively labeled protein reaching the cell body was determined as the ipsilateral minus the contralateral DRG and spinal cord and expressed both as total DPM as well as the ratio of cell body counts to counts remaining in the nerve.

\section{Statistical Analysis}

Several analyses were used to compare the accumulation at different time points. Because of the variation in the amount of injected radioactivity, we initially performed the analysis using the ratio of counts in the cell body to counts remaining in the nerve. These ratios were on the order of $10^{-3}$ to $10^{-5}$. Using analysis of covariance we assessed the relationship between the counts in the cell bodies and those remaining in the nerve in individual control and experimental animals and found that there was no statistically significant linear relationship between those counts at any single time point. We then performed analysis of variance using either TCA precipitable counts or the ratios of cell body to nerve counts with Bonferroni's procedure for multiple comparisons [13] to control for the multiple comparisons being made. Results from both analyses led to the same conclusions, but we found that analysis of the counts accounted for a greater proportion of the variability than did the analysis of the ratios. Therefore we have expressed the data as TCA precipitable disintegrations per minute of the DRG or spinal cord (ipsilateral minus contralateral), rather than ratios, in the figures presented here.

\section{Light Microscopic Autoradiography}

Under chloral hydrate anesthesia the animals were perfused through the heart with $0.1 \mathrm{M}$ phosphate buffer followed by $3 \%$ glutaraldehyde, $0.5 \%$ paraformaldehyde in $0.1 \mathrm{M}$ phosphate buffer pH 7.4 for approximately 1 hour. The L4, L5, and L6 DRGs and lumbosacral spinal cord were dissected out and kept in fixative an additional 2 hours at 4 degrees $\mathrm{C}$. The spinal cord was then cut into $2 \mathrm{~mm}$ segments, the tissue postfixed by $2 \% \mathrm{OsO} 4$ in the same buffer, dehydrated in ethanol and embedded in Polybed-Araldite mixture. Semithin (1 micron) sections were coated with Kodak NTB-2 nuclear track emulsion, exposed in the dark at 4 degrees $\mathrm{C}$ and developed in Kodak D-19 developer.

\section{RESULTS}

Radioactive counts began to appear in the DRG at 12 hours and the spinal cord 24 hours after ${ }^{3} \mathrm{H}$ NSP injection. In animals sacrificed immediately after injection or in animals in which the sciatic nerve was crushed between the injection site and the cell bodies prior to ${ }^{3} \mathrm{H}$ NSP injection no counts accumulate in the cell bodies of the sensory or motor neurons, suggesting that the labeled proteins reaching the

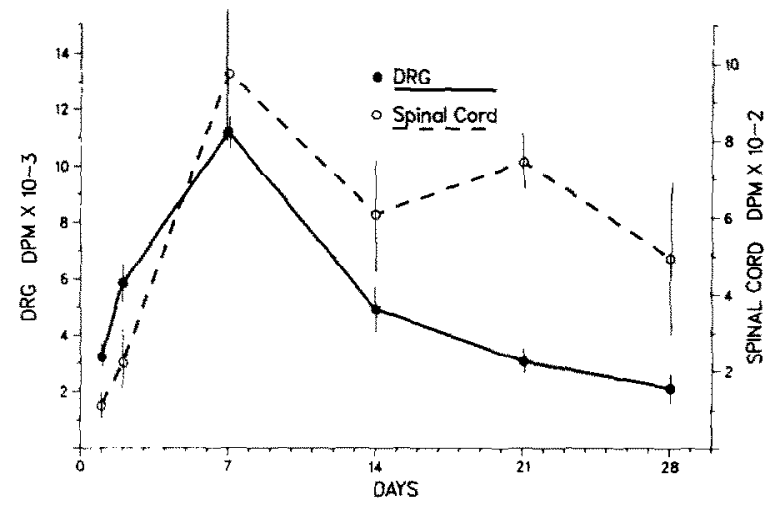

FIG. 2. The accumulation of TCA precipitable radioactivity in the pooled L4, L5 and L6 dorsal root ganglia (ipsilateral to ${ }^{3} \mathrm{H}$ NSP injection minus contralateral) and the lumbar ventral horn of spinal cord (ipsilateral minus contralateral punch samples) at 1 to 28 days after the intraneural labeling of endogenous nerve proteins with ${ }^{3} \mathrm{H}$ NSP. Values represent mean plus or minus SEM.

DRG and the spinal cord are transported via the nerve rather than being carried systemically.

To demonstrate that the localization of transported proteins in the DRG and spinal cord is in the cell bodies, we performed light microscopic autoradiography at an early (1 day) and later ( 5 day) time point. As demonstrated in Fig. 1 the radioactive grains in the DRG ipsilateral to the injection are located almost exclusively in the neuronal perikarya. Although a few axons have some grains, most of the axons passing through the DRG and the satellite cells are unlabeled. DRGs contralateral to the injected nerve had no visible labeling at similar times of exposure, in agreement with the biochemical data.

Similarly, the spinal cord at 1 day and 5 days show labeled motor neuron perikarya, while the surrounding neuropil and contralateral ventral horn neuronal perikarya are unlabeled. At 5 days grains are found surrounding the perikaryon compatible with labeled proteins in the dendritic tree of the motor neurons.

The quantitative analysis of the accumulation of radioactively labeled TCA precipitable proteins in the DRG cells is shown in Fig. 2. The increase in counts from 1 day to 2 days is statistically significant $(p<0.005)$ as is the increase from 2 days to 7 days $(p<0.005)$. Beyond 7 days the DRG counts drop significantly (comparing 7 days to 14 days $p<0.005$, comparing 14 days to 21 days $p<0.05$ ) and then appear to plateau at the lower level.

The accumulation of radioactively labeled TCA precipitable counts in the lumbar ventral horn is also shown in Fig. 2. Counts begin to appear at 24 hours after injection. The apparent increase in counts from 1 day to 2 days is not statistically significant $(p=0.6)$ while the increase from 2 to 7 days is significant $(p<0,001)$. Beyond 7 days the counts plateau; the

\section{FACING PAGE}

FIG. 1. Light microscopic autoradiography of ipsilateral ventral horn of spinal cord 1 day (A) and 5 days (B), and ipsilateral dorsal root ganglia 1 day (C) and 5 days (D) after intraneural injection of ${ }^{3} \mathrm{H}$ NSP. In all cases the radioactive grains are found almost exclusively in the perikaryon of the dorsal root ganglion neurons and motor neurons respectively. The DRG and spinal cord contralateral to the injection exposed for a similar length of time showed no significant grains above background. (A) $\times 686$ (B) $\times 634$ (C) $\times 704$ (D) $\times 634$ 
14 day and 21 day counts are not statistically different from 7 days, but the counts at 28 days are significantly reduced in comparison to 7 days $(p<0.05)$.

\section{DISCUSSION}

There are 2 findings of this study which merit further discussion. First, we have quantitated the accumulation of retrogradely transported labeled axonal proteins at the cell bodies of motor and sensory neurons. In each case the early accumulation at 24 hours of a small amount of labeled protein is followed by the subsequent accumulation of a larger amount of labeled protein before declining. This quantitative analysis of retrograde axonal transport will allow us in the future to determine whether there are alterations in that transport after nerve injury or in toxic states and should allow us to dissect out the role of retrogradely transported components in the biology of the neuron.

This technique does not allow us to definitively establish the velocity of retrograde axonal transport. The total accumulation in the cell bodies at any time point represents the balance between newly arriving labeled proteins and the degradation of labeled protein already in the cell bodies. In addition, the reutilization of labeled breakdown products in de novo protein synthesis may alter the shape of later portions of the curve. We have already published fluorographs of the pattern of radioactively labeled proteins which accumulate in the DRG [7]. Unlike anterograde transport, where the constellations of proteins transported at different rates are dramatically different [11], the major labeled bands at 1, 4 and 7 days appear similar (though we are currently undertaking quantitative gel scanning to determine whether some minor variations between those time points might be significant). This suggests that, at least in the DRG, there are not retrogradely transported "waves" of different proteins, but rather the differential accumulation over time of similar proteins.

The second finding of interest is the comparison between accumulation in the sensory neurons of the DRG and the motor neurons of the spinal cord. The injection site is not uniformly fixed in the sciatic nerve, but in general the distance from the injection site to the ventral horn is roughly twice the distance from the injection site to the DRG. The DRG counts are low at 1 day, increase significantly by 2 days, peak at 7 days and return to low levels by 14 days. The spinal cord in contrast still has low counts at 2 days, reaches a peak by 7 days, but remains at that high level for at least 2 weeks. The amount of counts in the spinal cord at 28 days is significantly less than that found at 7 days, but nonetheless is significantly greater than the number of counts found at 1 day.

There are three possible explanations for the differences. In part the differences between the curves must reflect the relative distances from the injection site to the cell bodies. While it would require a more detailed analysis of the curves to determine how analagous they are, that distance alone cannot be the entire explanation is suggested by the difference between the 14 day DRG (which is as low as the 1 day DRG) and the 28 day spinal cord (which remains significantly above the level of the 2 day spinal cord). This late plateau might be due to a second, late arriving wave of transported radioactively labeled proteins at the motor neurons. Alternatively the turnover and reutilization of labeled amino acids may be different in the two neuronal systems.

Retrograde axonal transport is one important means by which the neuronal perikaryon is informed of the state of the axon. Recent evidence using a number of different methods suggests that alterations in retrograde axonal transport may play a role in the development of toxic and metabolic neuropathies $[10,12]$. The ${ }^{3} \mathrm{H}$ NSP method may prove to be a useful way to further investigate that possibility, and to identify macromolecules which serve signal functions in the normal neuron.

\section{ACKNOWLEDGEMENTS}

The statistical analysis was performed by Dr. Judy Bromberg of VA HSR\&D. We wish to acknowledge the excellent technical assistance in histology and photography of Julie Staple and Barbara Rogers and the secretarial help of Carolyn White. The graphs were made by Dr. Ron Craig. This research was supported by VA Merit Review Grants to Dr. Mata and to Dr. Fink. A preliminary communication of this work was presented in abstract form at the meeting of the Society for Neuroscience in October, 1985

\section{REFERENCES}

1. Bisby, M. A. Functions of retrograde axonal transport. Fed Proc 41: 2307-2311, 1981.

2. Boyd, H., I. C. Calder, S. I. Leack and B. Milligan. $\mathrm{N}$-acylsuccinimides as acylating agents for proteins: synthesis, hydrolysis and aminolysis. Int J Pept Protein Res 4: 109-115. 1972.

3. Cragg, B. G. What is the signal for chromatolysis? Brain Res 23: $1-21,1970$

4. Fink, D. J. and H. Gainer. The use of a labeled acylating probe for the study of fast axonal transport, in vivo. Brain Res 177: 208-213, 1979.

5. Fink, D. J. and H. Gainer. Retrograde axonal transport of endogenous proteins in sciatic nerve demonstrated by covalent labeling in vivo. Science 208: 303-305, 1980.

6. Fink, D. J. and H. Gainer. Axonal transport of proteins: a new view using in vivo covalent labeling. I Cell Biol 85: 175-186, 1980.

7. Fink, D. J., D. Purkiss and M. Mata. Rapid retrograde transport of proteins in sensory neurons in rats. Brain Res 345: 394-397, 1985.

8. Grafstein, B. and D. Forman. Intracellular transport in neurons Physiol Rev 60: 1167-1283, 1980.
9. Hedreen, J. C. and G. C. Holm. Retrograde and anterograde axonal transport demonstrated by intracerebral injection of a labeled protein acylating agent. Brain Res Bull 7: 665-670, 1981.

10. Jakobsen, J. and Q. Sidenius. Early and dose dependent decrease of retrograde axonal transport in acrylamide intoxicated rats. I Neurochem 40: 447-454, 1983.

11. Lasek, R. J. and S. T. Brady. The axon: a prototype for studying expressional cytoplasm. Cold Spring Harbor: Symp Quamt Biol 46: 113-124, 1982

12. Miller, M. S, and P. S. Spencer. Single doses of acrylamide reduce retrograde transport velocity. $I$ Neurochem 43: 1401$1408,1984$.

13. Miller, R. G. Simultaneous Statistical Inference. New York: McGraw Hill, 1966, p. 15.

14. Tong. Y. S., A. Davis and J. P. Kitchen. N-succinimidyl propionate: characterisation and optimum conditions for use as a tritium labelling reagent for proteins. I Lab comp Radiopharmacol 20: 277-284, 1983.

15. Williams, L. R. and B. W. Agranoff. Retrograde transport of goldfish optic nerve proteins labeled by $\mathrm{N}$ succinimidyl $\left({ }^{3} \mathrm{H}\right)$ propionate. Brain Re's 259: 207-216, 1983. 